\title{
Einführung: Lebenswissenschaft, Globalisierung und künftige Geschichtlichkeit. Zur Aktualität von Plessners Philosophischer Anthropologie im Vergleich
}

Die Philosophie steht heute längerfristig vor drei großen Herausforderungen, zu deren kategorialer Artikulation Plessners Philosophische Anthropologie einen wegweisenden Beitrag zu leisten vermag.

Erstens haben sich die bio-ökologischen und bio-medizinischen Erfahrungswissenschaften des weiten Themas Leben unter dem Titel der Lebenswissenschaften (life sciences) bemächtigt, während gleichzeitig die Sozial- und Kulturwissenschaften wie auch die Geisteswissenschaften dieses Thema weitgehend aufgegeben haben oder sich in die Begleitung der Anwendungen biowissenschaftlicher und medizinischer Forschungen zurückziehen. So hilfreich neue Bio-Technologien zweifellos werden mögen, ihre Reduktion von Komplexität wird nur dann nicht das Ganze personaler Lebensführung bedrohen, wenn sie als Teile und Aspekte in das Lebensthema eingeordnet werden können. Die Analyse von bestimmten Teilen und Aspekten kann den ganzheitlichen Lebensprozess nicht ersetzen, sondern muss ihn auch in Zukunft voraussetzen können, wenn analytische Reduktion nicht seine Zerstörung bedeuten soll. Dafür ist die naturphilosophische Freilegung derjenigen Präsuppositionen wichtig, die die Bio-Lebenswissenschaften auch für ihre eigene Lebensführung und die Zukunft ihrer Forschung im Ganzen unterstellen, ohne sie selbst zum Gegenstand ihrer Forschung machen zu können. Würde man die analytisch nötigen Reduktionen der life sciences auf sie selber kausal anwenden, müsste man sie als geistig-kulturelle Unternehmungen schlichtweg abschaffen.

Zweitens: Die bisherige Globalisierung unterliegt dem neoliberalen Primat der von der Realwirtschaft abgekoppelten finanzwirtschaftlichen Verwertung aller möglichen Vermögen und Produkte einschließlich digitaler Kommunikationspotentiale. Diese Primatsetzung geht nicht nur mit ökologisch desaströsen Folgen einher, obgleich man Märkte ökologisch regulieren kann, sondern behindert vor allem die Entfaltung der Spezifik von politischen und soziokulturellen Austauschbeziehungen. In den probabilistischen Modellen zum Kauf von Finanzprodukten und in den betriebswirtschaftlichen Modellen kommt die Spezifik der Personalität von Lebensformen nicht mehr vor (siehe Herzog/ Honneth 2014). Das Politische, das im öffentlichen im Unterschied zum privaten Leben mündiger Bürger und Bürgerinnen entstehen kann, wird im Namen eines 
technischen Zwangs zur Transparenz unterlaufen, die privat und geheimdienstlich verwertet wird. Angehörige der biologischen Spezies homo sapiens sapiens gelten global gesehen nicht, wie es in den westlichen Verfassungen vorgesehen ist, als Potential für die Aktualisierung personaler Lebensformen in Gemeinschaft und Gesellschaft, wenn es etwa um sogenannte Flüchtlingsmassen geht. Umso wichtiger ist die sozial- und kulturphilosophische Rekonstruktion derjenigen Struktur- und Funktionsbedingungen, die spezifisch personale Lebensprozesse qualitativ ermöglichen.

Drittens: Verglichen mit der eingefrorenen Lage während des Kalten Krieges erfährt Europa seit 1989-92 eine Rückkehr zur Geschichte in dem ganzen Spektrum von „nachholenden Revolutionen“ (Habermas 1990) über die Neuordnung der „Imperien“ (Münkler 2008) bis zu „neuen Kriegen“ (Münkler 2010). Der Aufstieg Chinas und Indiens, die Überschuldung der USA und ihre Verletzbarkeit auf ihrem eigenen Territorium deuten auf einen Kampf zwischen monopolarer und multipolarer Weltordnung hin. Um sich geschichtsphilosophisch in dieser neuen Lage offenen Ausgangs gleichwohl orientieren zu können, wäre es wohl hilfreich, sich in dem Spektrum an Machtphänomenen von ihrer Pluralisierung bis zu ihrer Monopolisierung zurechtzufinden und zu verstehen, inwieweit das Außerwestliche in einem nicht-romantischen Sinne dem Westlichen anders und fremd bleiben könnte und inwieweit es längst verwestlicht ist.

Im Hinblick auf diese drei Herausforderungen werde ich hier in der Einführung an drei Fundierungsweisen in Helmuth Plessners Philosophischer Anthropologie erinnern, wodurch deren systematische Aktualität im Ganzen hervortreten kann. Es handelt sich 1. um ihre naturphilosophische, 2. sozial- und kulturphilosophische und 3. geschichtsphilosophische Fundierung von Lebensprozessen im Hinblick auf deren personale Spezifik. Diesen drei Fokussen sind auch die ersten drei Teile des folgenden Buchs gewidmet. Der vierte Teil vertieft das Verständnis dieser Philosophischen Anthropologie durch ihren Vergleich mit anderen Philosophien.

Fundierung heißt hier zunächst einmal Rekonstruktion der für das jeweilige Spektrum an Phänomenen, d. h. an qualitativen Erfahrungen, wesentlichen Ermöglichungsstrukturen. Diese Rekonstruktion erfordert eine phänomenologische Beschreibung und hermeneutische Deutung der Phänomene. Diese philosophischen Methoden werden aber nicht im Sinne dualistischer Vorurteile (entweder Materie oder Geist, entweder Physisches oder Psychisches, entweder Natur oder Kultur, entweder ungeschichtliche Konstante oder geschichtliche Ereignishaftigkeit) verwendet, sondern dialektisch im Sinne einer gleichzeitigen Konjunktion zwischen den Gegensatzgliedern. Die Einheit des jeweiligen Gegensatzes hybridisiert ihn zu einer Lebenseinheit, deren Widerspruch prozessiert. 
Die Verschränkung der Gegensätze zu ihrer Einheit geschieht jeweils durch ein Drittes.

\section{Die naturphilosophische Fundierung der personalen Lebenssphäre}

Die bekannteste Verschränkung aus dieser Philosophischen Anthropologie liegt in ihrer naturphilosophischen Fundierung der „Sphäre“ personalen Lebens durch eine „exzentrische Positionalität“ vor. Die personale Lebenssphäre wird, phänomenologisch anhand von „Menschen“ vorgestellt, im Unterschied und Zusammenhang mit der „Sphäre“ bereits bewussten Lebens, das phänomenologisch anhand von höher entwickelten „Tieren“ (Plessner 1975, 237-245, 288-293) beschrieben wird. Die Aufgabe der Naturphilosophie lautet aber insgesamt viel grundsätzlicher, Natur so neu zu differenzieren, dass in ihr personales Leben einschließlich seiner anorganischen und organischen Prozessbedingungen als wirklich möglich verstanden werden kann. Plessner praktiziert hier eine naturphilosophisch teilnehmende Beobachtung der biologischen Forschung. Deren empirische Erfahrungen zielen auf experimentell darstellbare und messbare Ergebnisse, die die Einrichtung quantifizierbarer Kontinua erfordern. Aber diese Resultate setzen voraus, dass man um die qualitative Irreduzibiltät der Phänomene und ihre wesentlichen Unterschiede untereinander in der Anschauung und deren Deutung insbesondere in der Anwendung der Ergebnisse weiß. Die Naturphilosophie expliziert dieses vorausgesetzte Anschauen und Verstehen der Phänomene (ebd., 34-36, 67) durch ihre „Kategorien“, die als „Formen der Übereinstimmung zwischen heterogenen Sphären, sowohl zwischen Denken und Anschauen wie zwischen Subjekt und Objekt“, die empirischen „Begriffe“ allererst „ermöglichen“ (ebd., 116). Die Naturphilosophie kann nicht - wie aber die Naturwissenschaft - eine Kausalerklärung leisten. Die Naturphilosophie zielt auf die irreduzible Qualität der Phänomene, d. h. ihre je verschiedene Wesensart, ab, wenn sie die drei folgenden Grenzfragen stellt:

Wie kann a) der Unterschied zwischen anorganischer und lebender Natur derart begriffen werden, dass - bei aller Gestaltkontinuität zwischen ihnen anorganische Zusammenhänge in spezifisch lebendige Phänomene funktional integriert werden? Für diese Frage entwickelt Plessner die Hypothese, dass Körper leben, insofern sie ihre eigene Grenze vollziehen. Sie können so in der Anschauung raumhaft (eigenräumlich) über sich hinaus und in sich hinein gehen, zeithaft (eigenzeitlich) sich vorweg und hinter sich her sein, indem sie sich gegenüber sie umgebenden Medien öffnen und schließen. Eben dies erwarte man als spezifische Qualität der Lebendigkeit in ihrer Anschauung. Körper 
als lebende entfalten - über ihre anorganische Beschaffenheit hinausgehend und an diese gebunden - einen Grenzübergang in das ihnen Äußere und von dort in das ihnen Innere zurück (siehe ebd., 99-105).

Freilich kann die Erfüllung dieser Anschauung spezifischer Lebendigkeit nur dem Scheine nach vorliegen, weshalb Plessner die phänomenologische durch eine hermeneutische Analyse erweitert. Unter welchen ontischen und ontologischen Prozessbedingungen des lebendigen Daseins, die sich unter dem Gesichtspunkt der Grenzhypothese verstehen lassen, wird diese Anschauung spezifischer Lebendigkeit zur Wirklichkeit (siehe ebd., 121-126, 171-180)? Wenn der Körper durch Vollzug seiner Grenze aus sich heraus über sich hinaus geht und von dort sich entgegen in sich zurückkehrt, dann „hebt“ er sich in diesem Grenzdurchgang „an“ und wird so „setzbar“: Er „positioniert“ sich, indem er nicht nur eine Stelle in der physikalischen Raumzeit ausfüllt, sondern sich von außerhalb und innerhalb seiner zu dieser Stelle verhält, sie als seinen Ort und seine Zeit „behauptet“ (ebd., 129). Insoweit ist jeder lebendige Körper „kein bloßes Ding, sondern ein Wesen“, ein „Fürsichsein“ (ebd., 131), ohne Bewusstsein oder Selbstbewusstsein aufweisen zu müssen. „Ein Ding positionalen Charakters kann nur sein, indem es wird; der Prozess ist die Weise seines Seins“ (ebd., 132). In ihm können die sich widersprechenden Momente des lebendigen Dinges so in eine Kontinuität von Phasen verteilt werden, dass sie sich im Ganzen integrieren lassen.

Der dynamisch gerichtete Charakter dieses Prozesses, sein Entwicklungscharakter, wird als der fortwährende „Ausgleich einer Unfertigkeit“ in seiner „Selbstlenkung“ verstanden, die keiner von außen kommenden „Entelechie“ oder „Präformation“ (Plessner 1975, 142) bedarf. Dies ist eine klare Absage an jede vor-kantische Ontologie und jeden romantischen Neovitalismus. Dafür muss aber eine Art und Weise von schon vorbewusster Rückbezüglichkeit des lebendigen Körpers angenommen werden, die ein „systemhaftes Ganzes“ darstellt (ebd., 159). Diese „Funktion der Mitte“ in dem in sich zurücklaufenden Prozess ist „als die wirkliche Möglichkeit des Körpers oder sein Vermögen (Potenz) real“ (ebd., 161f.). Von solcher „Selbstregulation“ zeugen „Restitutionsphänomene“ und „Adaptationsphänomene“ (ebd., 164). Der lebendige Körper löst sich nicht in seine Teile, die Organe, auf, sondern hat sie zugleich als Mittel in seiner Potentialität und Aktualität im Ganzen. „Erst als Einheit von Zweck und Mittel ist der lebendige Körper Ganzheit oder autonomes System“ (ebd., 171). In der Anschauung begegnen solche Phänomene raumhaft als solche, die sich in einer „Entfaltung“ befinden, und zeithaft als solche, die sich selber vorweg und daher hinterher sind. „In Rückbindung von der Zukunft her steht der lebendige Körper, ihm selber vorweg, d. h. Zweck, seinem dauernden Überge- 
hen vom Noch- nicht ins Nicht- mehr entgegen und beharrt“ (ebd., 180). Das Differential seiner konkreten Gegenwart besteht in einem Augenblick der Einheit von Zukunft und Vergangenheit: „Seine Gegenwart ist jene Aktualität, die nicht mehr im unversöhnlichen Gegensatz zur Potentialität gedacht werden muss, sondern Potentialität zur Voraussetzung hat: erfüllte Potentialität“ (ebd.).

Wie lässt sich b) das Zusammenspiel zwischen den Organisationsformen, also den Gliederungen lebendiger Körper nach innen und von innen her, und den Positionsformen, also den struktur-funktionalen Möglichkeiten dieser Körper, sich in einer Umwelt nach außen und von außen zurück zu positionieren, verstehen? Diese Frage ergibt sich allererst aus Plessners Zugang zur Lebendigkeit von Körpern durch die Hypothese, sie könnten ihre eigene Grenze realisieren. Dieser Zugang hat den forschungsstrategischen Vorteil, dass er die früheren Organismus-Modelle des Lebens immanent in die anderen Lebensmodelle von der Interaktion des Organismus mit seiner Umwelt überführen kann:

In seinen Organen geht der lebendige Körper aus ihm heraus und zu ihm zurück, sofern die Organe offen sind und einen Funktionskreis mit dem bilden, dem sie sich öffnen. Offen sind die Organe gegenüber dem Positionsfeld. So entsteht der Kreis des Lebens, dessen eine Hälfte vom Organismus, dessen andere vom Positionsfeld gebildet wird. (Ebd., 191f.)

Stofflich-energetisch gesehen kommt nur dem Lebenskreis „Autarkie“ zu, während sich die „Autonomie“ des Organismus durch Heteronomie vermitteln muss: „Der Organismus ist Einheit nur als durch Anderes, als er selbst ist, in ihm vermittelter Körper, Glied eines Ganzen, das über ihm hinausliegt“ (ebd., 195).

Das Spektrum des Zusammenspiels der Organisations- und Positionalitätsformen wird in den Biowissenschaften evolutionstheoretisch durch Anpassung und Selektion erklärt. Welches naturphilosophische Vorverständnis kann hier nicht nur empiristische Missverständnisse, sondern auch den politischen Missbrauch solcher Begriffe kritisieren? Versteht man „Anpassung“ nur als ein $a k$ tuales Geschehen, das der Organismus wie ein „bloßer Spielball aller Kräfte“ unentwegt zu leisten habe, dann grenzt das Zusammenspiel an ein Wunder, weil es eine strukturale und funktionale „Angepasstheit“ im Vorhinein durch Potentialitäten voraussetzt. Man dürfe hier nicht, was man für einen ,anthropoklinen Fortschrittsprozess“ halte, in die Anpassung hineinprojizieren (Plessner 1975, 202). Gehe man umgekehrt von einer „ausschließlichen Angepasstheit“ aus, hätte der Organismus in der konkreten Gegenwart des Positionsfeldes keinen Kampf und keinen Schutz mehr nötig. Er könnte sich wie ein „Solipsist“ verhalten. Das Wechselspiel zwischen potentieller Angepasstheit und aktualer Anpassung ergebe sich vielmehr daraus, dass sich der Organismus im Positionsfeld zugleich an dessen „Peripherie“ und wie ein „exzentrischer Mittelpunkt“ 
verhält. Damit entfallen alle metaphysischen Annahmen über die „absolute Immanenz" oder die „absolute Transzendenz“ (ebd., 203) des Positionsfeldes für den Organismus. Nicht nur „passive Anpassung“, auch „Selektion“ wird üblicher Weise nach dem Modell des „Kampfes ums Dasein“ und der „Zuchtwahl“ von außen an das Leben herangetragen. Aber Leben stellt in anderem Sinne bereits in sich ein „notwendiges Versäumnis seiner Möglichkeiten und darin Selektion“ (ebd., 206), d. h. einen „kategorische[n] Konjunktiv“ (ebd., 216), dar.

Der Grenzübergang des lebendigen Körpers zu den ihn umgebenden Medien ermöglicht ein Spektrum zwischen seiner Öffnung und Schließung gegenüber der Umwelt. Folgt seine Organisation primär einer offenen Form, so wird er in all seinen Lebensäußerungen „unmittelbar seiner Umgebung eingegliedert“ und zu einem ,unselbständigen Abschnitt des ihm entsprechenden Lebenskreises“ (ebd., 219). Diese offene Organisationsform wird phänomenologisch anhand von Pflanzen vorgestellt. „Geschlossen ist diejenige Form, welche den Organismus in allen seinen Lebensäußerungen mittelbar seiner Umgebung eingliedert und ihn zum selbständigen Abschnitt des ihm entsprechenden Lebenskreises macht“ (ebd., 226). Die geschlossene Organisationsform begegnet phänomenologisch in der Anschauung von Tieren. Die Schließung nach innen kann in dem Spektrum zwischen dezentral (verschiedene Funktionszentren) und zentral (durch ein zentralnervöses Organ) erfolgen (siehe ebd., 241). Diese nervöse Vermittlung des Antagonismus zwischen sensorischen und motorischen Organen im Inneren ermöglicht verschiedene Funktionseinheiten in der äußeren Positionierung (siehe ebd., 244). Die eher dezentral organisierte Zuordnung zwischen Reiz und Reaktion öffnet das Lebewesen für die Erfahrung „komplexer Qualitäten“ (ebd., 264) in seiner Umwelt, ohne dass es sich in dieser als ein Subjekt bewusst werden könnte. Die mehr zentrische Zuordnung zwischen Reiz und Reaktion vermittelt die Antwort auf die Verhaltensfrage durch eine „Unterbrechung“, d.h. durch einen Hiatus (raumhaft) und eine Pause (zeithaft), im Zentralorgan (ebd., 245, 260). Mit dieser Abhebung des Zentralorgans vom Gesamtkörper, zu dem es in der Organisation doch gehört (siehe ebd., 243), korrespondiert in der Positionierungweise die spontane, ein „handelndes Subjekt“ ausmachende Kontaktaufnahme in seinem Positionsfeld: Es eröffnet sich ihm eine, zwischen „Merken“ und „Wirken“ (ebd., 232) „,bewusst“ werdende, „dingliche Gliederung der Umwelt“ (ebd., 252), zu der auch soziale „Mitverhältnisse“ (ebd., 306-308) der leiblichen „Koexistenz“ (ebd., 259) gehören. Diese organismische Existenz lebt kurz gesagt in einer zentrischen Positionalitätsform, die anhand höher entwickelter Tiere vorgestellt wird. 
Wie lässt sich c) der Zusammenhang zwischen den „exzentrischen“ und den „konzentrischen“ Potenzialen, sich in einer Welt zu positionieren, verstehen Plessner 1975, 294)? Die Frage nach dieser Verschränkung, die spezifisch personales Leben ermöglicht, stellt sich mit der exzentrischen Positionalität (ebd., 291f.). Konzentrik heißt, dass das Zentrum der Positionierung (nicht das Zentrum des Organismus) in der funktionalen Mitte zwischen dem Organismus und seiner Umwelt liegt, z. B. da, wo der Hund das Bällchen zum Apportieren gerade schnappt. Exzentrik bedeutet, dass die Verhaltensbildung raumhaft von außerhalb dieses leiblichen Zentrums und zeithaft aus ihm versetzt erfolgen kann. Wie ist dies möglich? - Nun dadurch, dass diese Lebewesen als Personen aus ihren leiblich-konzentrischen Einheiten mit der Umwelt heraustreten und in Relationen $\mathrm{zu}$ anderen Personen eintreten, von denen her sie sich $\mathrm{zu}$ sehen, $\mathrm{zu}$ hören, zu fühlen erlernen. Auf diesem Wege finden sie einen Abstand zu ihrer natürlichen Umwelt. Sie können diese in Welt, d. h. als „Außenwelt“, „Innenwelt“ und „Mitwelt“ (ebd., 293-304), rahmen. In dem exzentrischen Weltrahmen nimmt die Einrichtung von leiblich nötiger Umwelt einen Aufgabencharakter an. Was habituell an natürlicher Eingespieltheit nötig ist, gilt es erst künstlich durch Kultur und Technik, d.h. auf dem Wege „natürlicher Künstlichkeit“ (ebd., 309), herzustellen. Was intentional hier und heute wie selbstverständlich eine Erfüllung gewährt, braucht geschichtlich den Umweg über die Erfindung und Entdeckung von stabilisierbaren Vermittlungen, d. h. eine ,vermittelte Unmittelbarkeit“ (ebd., 321). Was die Bejahung eines - gerade für Personen als Mitglieder einer geistigen Mitwelt - widrigen und sterblichen Lebens im Letzten ermöglicht, ist ein über sie hinausgehender „utopischer Standort“ (ebd., 341), der selber zwischen dem Nichts und der absoluten Transzendenz geschichtlich schwankt.

In der exzentrischen Positionalität besteht einerseits die nötige Möglichkeit, das Verhalten über die inter-personalen Relationen hinausgehend immer weiter $\mathrm{zu}$ ex-zentrieren. Andererseits bleibt aber zugleich die intra-personale $R e$ zentrierung auf die leibliche Konzentrik zwischen Organismus und Umwelt nötig, denn auch Personen werden als Lebewesen keine reinen Geister. Die Naturphilosophie endet also nicht in einem fertigen Verhaltensrepertoire, sondern in dem Bruch (ebd., 292f.) zwischen Konzentrik und Exzentrik, der personales Leben in geschichtsbedürftige Verschränkungen führt. Es ist diejenige Lebensweise, die sich zu der Aufgabe wird, immer neu Exzentrik und Konzentrik im positionalen Vollzug verschränken zu müssen, ohne dafür eine endgültige Lösung haben zu können, obgleich sie hier und jetzt eine zu vollziehen hat.

Den drei Grenzfragen aus Plessners Naturphilosophie entsprechen aktuelle Herausforderungen: Die Synthetische Biologie unterläuft die Grenzfrage zwi- 
schen anorganischer und lebendiger Natur, indem sie auf die zellulare und interzellulare Molekülebene wechselt, was methodisch Erkenntnis- und Handlungsgewinn verspricht. Aber dieser Gewinn setzt voraus, dass er sinnvoll in die ganzheitliche und qualitative Lebensführung von Personen und deren „Vorbedingungen“ (ebd., 301) integriert werden kann. Die Ökologie wirft am anderen, vergleichsweise makroskopischen Ende des Spektrums vom Lebendigen die Frage nach der funktionalen Integration der verschiedenen Lebensprozesse im Sinne der Nachhaltigkeit auf. Wir konnten hier nicht die vielfältigen Potentiale zur Aktualisierung des Zusammenspiels zwischen Organisations- und Positionalitätsformen (a-zentrisch, dezentral, zentrisch, exzentrisch) durchgehen, aber Plessner behandelt sie nach der Maxime der „Gleichberechtigung“ (ebd., 202) aller Lebensformen, die sich ökologisch lesen lässt. Zwischen der synthetischbiologischen und ökologischen Frage liegen die aktuellen Fragen der vergleichenden Verhaltensforschung und Neurobiologie. Das Modell von der Exzentrierung und Rezentrierung der Verhaltenspotentiale lässt sich für das Vergleichsproblem von Menschen mit anderen Primaten und das Problem des Überschusses von Metarepräsentationen in den hirnphysiologischen Korrelaten von personalen Phänomenen fruchtbar machen (Krüger 2010).

\section{Die sozial- und kulturphilosophische Fundierung personaler Lebensformen}

Im Hinblick auf die Fundierung personaler Lebensformen gilt es, systematisch zu verstehen, wie die naturphilosophisch freigelegte Mitwelt in ihrer soziokulturellen Spezifik philosophisch fassbar wird. Was ermöglicht kategorial nun sozial- und kulturwissenschaftliche Erfahrung in einem qualitativen Sinne? Humane Lebewesen, erneut exemplarisch als Phänomene für die personale Lebenssphäre genommen, werden in Rollenkörper hineingeboren und sterben in solchen, die ihnen vorhergehen und sie überdauern. Solche Lebewesen erlernen, aus sich herausgehend, in diesen soziokulturellen Körpern eine Exzentrierung zur Person, die so Distanz zu ihrem Leib gewinnt und ihn als Körper haben kann. Und sie erlernen auf dem Rückweg eine Rezentrierung der Person auf ihren Leib, der so habitualisiert wird. Eine Person entsteht elementar durch ihre Selbstverdoppelung in die nach außen für andere öffentlich zugängliche Person, von der sie auf sich als private Person zurückkommt. Dieses öffentlichprivate „Doppelgängertum“ entfaltet Plessner in seinem Spätwerk als „Gelenk“ und „Nahtstelle“ zwischen Individuum und Gesellschaft (Plessner 1985h, 227f.; Plessner 1983e, 199-205). Dort geht er auch der politisch repräsentativen Ausgestaltung öffentlicher Rollen nach dem Modell des theatrum mundi und der 
spezifisch modernen Funktionalisierung von Rollen zu Leistungen in gesellschaftlichen Handlungsbereichen nach.

Diese Fundierung besteht sozial im Kern aus einer Theorie des sich Hineinspieles in und des sich Herausspielens aus Personenrollen, so dass man durch Identifikation in und auf Distanz mit diesen Rollen spielen kann (siehe Plessner 1982a u. 1982i). Homo ludens, derjenige Mensch also, dessen Wesen in solchem Spielen besteht, um Johan Huizingas allerdings kulturgeschichtlichen Buch-Titel aufzugreifen, existiert elementar dadurch, dass er Verhaltensdyaden zwischen Lebewesen trianguliert, also in Dreier-Beziehungen überführt, die als solche im Unterschied von und Zusammenhang mit anderen DreierBeziehungen verstanden werden. Dies erfordert kulturell Symbole, also dreigliedrige Zeichen, die als solche im Unterschied $\mathrm{zu}$ und Zusammenhang mit anderen Symbolen aufgefasst werden. Das Besondere an Plessners Zugang besteht darin, dass er den falschen Gegensatz zwischen sprachlichen und nichtsprachlichen Zeichen unterläuft, indem er die intermodal möglichen Einheiten der verschiedenen Sinnesmodalitäten praktisch versteht. Seine wichtigste dreigliedrige Symbolfunktion reicht von der aisthetischen „Thematisierung“ über die sprachliche „Präzisierung“ bis zur wissenschaftlich-technischen „Schematisierung“ (Plessner 1980b, 87, 154, 276) personalen Verhaltens.

Das Hauptproblem moderner Gesellschaften besteht darin, dass sie nicht als die Verallgemeinerung oder Hegemonie einer bestimmten Gemeinschaftsform begriffen werden können. Indem man einen familien- oder sachähnlichen Wertemaßstab einführt, was für bestimmte Aufgaben der Reproduktion und Unternehmung sinnvoll ist, gibt es immer gleichzeitig das für diesen Maßstab Andere und Fremde, das von ihm irgendwie Abweichende (siehe Plessner 1981b, 45-57). Die vollständige Vergemeinschaftung personalen Verhaltens widerspricht aber denjenigen Ambivalenzen, die in der leiblich unvertretbaren Individualisierung von Personenrollen entstehen, darunter zwischen Verhaltenheit und Scham einerseits, Geltungsdrang und Exponiertheit andererseits (ebd., 6376), oder zwischen Sucht und Leidenschaft für Anderes und Fremdes (siehe Plessner 1983b u. 19831). Plessner setzt gegen einseitige Vergemeinschaftungen auf den Maßstab einer zivilen Gesellschaft, die offen ist für Interaktionen unter einander Unbekannten, Anderen und Fremden, die auch künftig einander unbekannt, anders und fremd bleiben können. Die Attraktion dieses gesellschaftlichen Ethos des Spiels und der Grazie besteht nicht darin, den Individuen ihre Gemeinschaftlichkeit und Privatheit zu nehmen, sondern darin, die Individuen nicht derjenigen Privatheit und Gemeinschaft $\mathrm{zu}$ opfern, in die sie durch lebensgeschichtliche Zufälle hineingeboren worden sind. In Gesellschaft können ihnen noch unbekannte, andere und fremde Partizipationsmöglichkeiten ent- 
stehen. Die Politik steht damit vor der Aufgabe, durch das Medium des Rechts die Ansprüche auf Gemeinschaftlichkeit und Gesellschaftlichkeit der Individuen in einem öffentlichen Prozedere zu verschränken (ebd., 115-118, 131-134).

Rollenspiele haben individuell-leibliche Vollzugsgrenzen und hängen historisch von den gerechten Partizipationsmöglichkeiten an dem soziokulturellen Relationengefüge von Personenrollen ab. Die Grenzen des Rollenspiels werden im Lachen und Weinen von Kindesbeinen an erfahren. Der Hiatus zwischen der Exzentrierung und der Rezentrierung tritt insbesondere in diesem ungespielten Verlust personaler Selbstbeherrschung hervor. „Der Mensch verfällt ihnen, er fällt - ins Lachen, er lässt sich fallen ins Weinen. Er antwortet mit seinem Körper als Körper wie aus der Unmöglichkeit heraus, noch selber eine Antwort finden zu können“ (Plessner 1982f., 234f.). Springt in der exzentrierenden Richtung des Lachens die Verkörperung der Person in mehrsinnige Welten hinaus, ohne dass ihr der Leib noch nachkommen könnte, lösen sich in der rezentrierenden Richtung des Weinens die Welthorizonte in eine Leiblichkeit auf, die im Organismus zusammensackt. „Geöffnetheit, Unvermitteltheit, Eruptivität charakterisieren das Lachen, Verschlossenheit, Vermitteltheit, Allmählichkeit das Weinen“ (ebd., 368; vgl. Prütting 2013). Wolfgang Iser hat seine literarische Anthropologie im Anschluss an Plessners o.g. utopischen Standort entwickelt (Iser 1991) und ist grandios dem Ersticken des von Plessner erhellten Lachens und Weinens in der Rezeption von Samuel Becketts Stücken nachgegangen (Iser 1979).

Der aktuelle Kern von Plessners sozial- und kulturphilosophischer Fundierung besteht in der Einsicht, dass personales Leben wesentlich durch das öffentlich-private Doppelgängertums für all seine Mitglieder konstituiert wird. Dem widerspricht die gegenwärtige Auflösung der Unterscheidung zwischen Privatem und Öffentlichem in der wirtschaftlichen und staatlichen Monopolisierung von insbesondere digitalen Kommunikationspotentialen. Ohne die Selbstverdopplung von Personen ist es jedoch nicht möglich, die gesellschaftliche Pluralisierung auszuleben und gleichzeitig an der leiblichen Unvertretbarkeit der Individuen festzuhalten. Die Selbst-Relation in der Stimme des Gewissens einer Person ist nicht durch öffentlichen Dialog zu ersetzen. Ebenso wenig kann das individuelle Selbstgespräch an die Stelle gemeinschaftlicher und gesellschaftlicher Kommunikationsformen treten. Diese drei Phänomene können sich nur aneinander ausdifferenzieren und gegenseitig stützen, wenn das Doppelgängertum real praktizierbar ist (siehe Krüger 1999, 4. u. 5. Kap.). Anderenfalls können in der Tat „Sozialpathologien“ und „Psychopathologien“ um sich greifen (siehe Jaeggi 2014). Ich verstehe die politische Aufgabe der Kritik vom Standpunkt des Zusammenhangs zwischen je individueller Personalität und 
den ihr nötigen Gemeinschafts- und Gesellschaftsformen. Gesa Lindemann hat die Produktivität von Plessners Ansatz für eine mehrdimensionale Auffassung des Sozialen ausgearbeitet (Lindemann 2014). Andreas Heinz hat überzeugend gezeigt, wie die Psychiatrie und Psychotherapie in ihrer klinischen Praxis nicht nur Außen- und Innenwelt, sondern auch die Mitwelt voraussetzen muss. Sowohl Über-Exzentrierungen, denen eine ausgleichende Rezentrierung fehlt, als auch Über-Rezentrierungen, denen eine entsprechende Exzentrierung mangelt, können zu spezifisch personalen Krankheiten führen (Heinz 2014).

\section{Plessners geschichtsphilosophische Fundierung personalen Lebens}

Plessners Geschichtsphilosophie steht im Zeichen der Unergründlichkeit des Menschen und ist am wenigsten rezipiert worden, obgleich sie die auffälligste Konstante in seinem Lebenswerk darstellt. Dieses Schweigen sagt eher etwas über die Ideologiebedürftigkeit seiner Zeitgenossen als über ihn aus. Plessner war, deutlich in seiner Habilitationsschrift (1920), Agnostiker im Sinne der Kantschen Art und Weise, zu den Antinomien der Vernunft Stellung zu nehmen. Verwechsele man nicht die „Annäherung ans Ideal“, in symbolischer Hinsicht zu verfahren, mit der schematischen „Ausführung einer Idee“, dann bilde die „Würde den Boden des spezifischen Wertkreises der Philosophie“, allerdings die Würde für „endliche vernünftige Wesen“ als deren Lebenshaltung (Plessner 1981e, 270).

Die Durchführung dieses Normativs der Bewahrung personaler Würde für das menschliche Individuum wechselt in Plessners Werken je nach Thema und Kontext: In seinem Buch über die Grenzen der Gemeinschaft von 1924 plädiert er für eine Bewahrung der Würde durch die Situierung des Individuums in Gemeinschaft und Gesellschaft statt in entweder Gemeinschaft oder Gesellschaft. „Würde hat nichts mit Ehre zu tun“, d. h. mit der Identifikation mit einem Stand, einer Klasse oder Profession: „Würde betrifft stets das Ganze der Person, den Einklang ihres Inneren und Äußeren, und bezeichnet jene ideale Verfassung, nach der die Menschen streben, die aber nur wenigen verliehen ist“ (Plessner 1981b, 75f). In seinem Buch Macht und menschliche Natur von 1931 schließt Plessner direkt an Georg Mischs Prinzip von der Unergründlichkeit des geschichtlichen Lebens aus Diltheys Lebensphilosophie an (Schürmann 2011). Die verbindliche Übernahme dieses Prinzips bedeutet zunächst einmal einen schöpferischen Verzicht: „In dem Verzicht auf die Vormachtstellung des eigenen Wert- und Kategoriensystems gibt sich der europäische Geist den Horizont auf die ursprüngliche Mannigfaltigkeit der geschichtlich gewordenen Kulturen 
und ihrer Weltaspekte als einer offenen, unbegrenzten, durch keinen,Weltgeist planvoll gebundenen Mannigfaltigkeit frei“ (Plessner 1981d, 185). Aber in diesem Verzicht wird auch eine gemeinsame Zukunft ermöglicht: „Denn der Begriff des Menschen ist nichts anderes als das ,Mittel', durch welches und in welchem jene wertedemokratische Gleichstellung aller Kulturen in ihrer Rückbeziehung auf einen schöpferischen Lebensgrund vollzogen wird“ (ebd., 186). Im Spätwerk kommt dann auch die Transformation der negativen Theologie vom deus absconditus in den homo absconditus hinzu (Plessner 1983m, 134; Plessner 1983h). So wie man aus den Religionskriegen die Unergründlichkeit Gottes gelernt habe, könne man aus den Ideologiekriegen des 20. Jahrhunderts die Unergründlichkeit des Menschen erlernen.

Aber wie kann dieses Normativ, die Unergründlichkeit personalen Lebens $\mathrm{zu}$ achten, strukturell und funktional ermöglicht werden? Indem man jede Form der Machtbildung der Gewaltenteilung unterwirft und Medien des Ausgleichs zwischen den getrennten Gewalten einrichtet. Wenn sich Plessner in seinem Buch Die verspätete Nation (Erstausgabe 1935) für etwas interessiert, dann sind es die Gewaltenteilungen, die Medien des Rechts und der Öffentlichkeit, die aus den Erfahrungen von Kriegen und Bürgerkriegen die Konsequenzen ziehen, vom Alten Rom über die Gewaltenteilung zwischen Kaiser und Papst im Mittelalter bis zur Einrichtung der Grundrechte in der englischen, niederländischen, amerikanischen und französischen Revolution. Drei Jahre vor dem Münchner Abkommen schlägt er „unzeitgemäß“ vor, die deutschsprachigen Völker Europas könnten ihre föderalen und ökumenischen Traditionen für die „Vereinigten Staaten von Europa“ (Plessner 1982e, 50; siehe Koselleck 2000) stark machen, statt einen einzigen Nationalstaat und dessen imperiale Rolle anzustreben.

Was steht nun aber dieser Institutionalisierungsweise der Unergründlichkeit des Menschen im Wege? „Hochkapitalismus“ (Plessner 1982e, 97) ist kein Kapitalismus der freien Konkurrenz. Im Hochkapitalismus werden Wissenschaft und Technik durch eine Verwertungsökonomie verbunden, die auf die Erzeugung neuer Märkte für neue Bedürfnisse ausgerichtet ist. Die Umstellung der ökonomischen Produktion auf eine naturwissenschaftliche Methodik führe im Verhältnis zur äußeren Natur dazu, dass die Arbeit als Reichtum schaffende Quelle reduziert und tendenziell ersetzt werden kann. Neu sei aber vor allem die Veränderung des Verhältnisses zu der dem Menschen eigenen Natur. Im Hochkapitalismus werden neue „Lebensmächte“, so Plessners Kategorie von 1935, produziert. Paradigmatisch für eine „Lebensmacht“ (ebd., 98-100) steht bei Plessner die naturwissenschaftliche Definition von Krankheit und Gesundheit, die durch Einrichtungen der sozialen Hygiene, eines neuen Verhältnisses zwischen Ärzten und Patienten und neuer Märkte der Pharmaindustrie institutiona- 
lisiert wird. Plessner entdeckt hier das Thema, das Foucault später auf andere Weise unter dem Titel der Produktivität von Biomächten rekonstruiert hat (siehe Krüger 2001, 1. Kap.; Krüger 2009a, 1. Kap.).

Wie das Beispiel der sozialen Hygiene zeigt, handelt es sich bei Lebensmächten nicht nur um ökonomisch, sondern auch um politisch verwertbare Innovationen. Obgleich Plessner nie ein Marxist in der Lösung war, so gibt er doch dem Marxismus in der Analyse Recht: Der Preis für die neuen produktiven Möglichkeiten des Hochkapitalismus bestehe darin, dass diese Ökonomie den Handlungsbereich des Wirtschaftens überschreite, auf andere Handlungsbereiche übergreife, indem die Autonomien der Rechte, Wissenschaften, Künste, Politik unterlaufen werden, bis die ganze Gesellschaft strukturell dem „Geist und Zwang grundsätzlicher Überbietung jedes erreichten technischen Standes“ (Plessner 1982e, 97f.) unterworfen wird. Die Auflösung der modernen Funktionsteilung zwischen sachlogischen Handlungsbereichen, wie sie Max Weber angedacht habe, führe in ein „System der ungehemmten Expansion“ (ebd., 186). Dem „Zuwachs an Macht“ durch rein instrumentelle Rationalisierung „entspricht kein Zuwachs an Freiheit. Er vertausendfacht den menschlichen Wirkungsgrad, versklavt aber den einzelnen an eine lebens- und freiheitsfeindliche Gesellschaftsordnung“ (ebd., 100). Umso weniger kann für Plessner die kommunistische Verstaatlichung der Ökonomie die Lösung sein, denn sie führt zu einer weiteren Machtkonzentration von ökonomischer und politischer Macht. Real betrifft sie den Nationalstaat, der in Relation zu anderen Nationalstaaten in der Regel einem Imperium angehört oder dieses selbst anstrebt.

Plessner denkt die Konsequenzen der expansiven Logik des Hochkapitalismus als den Prozess der Ablösung Europas von Europa, der Ablösung, rechnet man die neue Welt der USA hinzu, des Westens vom Westen. „Europa siegt, indem es entbindet“ (Plessner 1981d, 164). Was ist mit der Ablösung des Westens vom Westen gemeint? Zunächst seien die Resultate, dann aber auch die Methoden des Westens vom Geist ihrer Erzeuger ablösbar. Die Ablösbarkeit ergebe sich aus dem formal rechenhaften und funktionalen Charakter der westlichen Verfahren. In ihnen werde von konkreten personenbezogenen Zwecken und Mitteln zugunsten anonymer und allgemeiner Schemata in Operationalisierung und Automatisierung abstrahiert. Diese allgemeinen Anwendungsmöglichkeiten setzen eine Art und Weise von Theorie voraus, die sich ihrerseits nochmals vom Ethos ihrer Erzeuger unterscheiden lasse:

Diese Expansion ist nur möglich gewesen und praktisch unbegrenzt, weil die Ergebnisse der Wissenschaft in ihren Anwendungsmöglichkeiten aufgehen und die Handhabung der Apparate und Maschinen zwar an das Verständnis der Theorie, aber nicht an das Verständnis für das humanistische Ethos der Theorie gebunden ist. Autofahren und Auto- 
bauen ist zweierlei. Aber um ein Auto zu bauen, braucht man keine humanistische Tradition in sich zu haben, braucht man weder Faust zu sein noch Faust zu verstehen. Auf der Ablösbarkeit der technischen Anwendung von der Theorie beruht der industrielle Fortschritt, auf der Neutralisierbarkeit der Theorie gegen das sie tragende Ethos, [...] beruht die Europäisierung der Erde. (Plessner 1982e, 38)

Ablösbarkeit vom Westen heißt nicht nur, dass sich außerwestliche Kulturen die Resultate, sodann die Methoden des Westens aneignen, sondern dass sie sie auch modifizieren und verändern können. Die Frage, wie sie all das können, hängt natürlich von der Art und Weise dieser außerwestlichen Kulturen ab, insbesondere davon, ob sie bereits Achsenkulturen waren. Plessner gibt 1935 das Beispiel Japans, heute stehen dafür China und Indien:

Nur um den Preis seiner Mechanisierung und Instrumentalisierung erobert der Europäismus die Welt. Diese seine Übertragbarkeit auf nichtchristliche Kulturen wird ihm zum Schicksal. Äußerlich dadurch, dass er sich Konkurrenz auf Konkurrenz großzieht, innerlich dadurch, dass die Leistungen europäischen Geistes von ihm selbst unabhängig werden. (Ebd., 39)

Der Hochkapitalismus stellt alle tradierten, sowohl westlichen als auch nichtwestlichen Kulturen, deren Welt- und Selbstverständnis, grundsätzlich in Frage. Was können sie wozu mit seinen Resultaten und Methoden anfangen? Er produziert eine Serie immer neuer Positivitäten, die für ökonomische und politische Verwertungen immer hier und jetzt markiert und monopolisiert werden können. Insofern steht er der gewaltenteiligen Institutionalisierung von Unergründlichkeit entgegen. Aber als Prozess vernichtet der Hochkapitalismus auch immer neu die gerade markierten Positivitäten. Wenn er produktiv bleiben soll, dann bleibt er in seinen Rekombinationen darauf angewiesen, dass aus den verschiedenen Handlungsfeldern noch produktive Leistungen kommen. Für Plessner sind soziokulturell produktive Leistungen im modernen Sinne an den Status einer Personalität gebunden, die auf keine Positivität eingeschränkt wird. Insoweit bleibt ihm die Produktivität des Hochkapitalismus von den gewaltenteiligen Institutionen der Unergründlichkeit abhängig.

Die Versuche, dem Hochkapitalismus einen politischen Ordnungsrahmen zu geben, müssen aber nicht in demokratischer Richtung ausschlagen. Wie Plessner 1931 in seiner Diskussion mit Carl Schmitt zeigt, ist eine Autonomisierung der Politik möglich. Sie kann entlang der Dichotomie von Freund und Feind Angst bannen, entfesseln und steigern. Einerseits verspricht sie Vertrautheit und Nähe gegen die Offenheit der Welt, die Reduktion der Komplexität von Welt auf eine klare Anpassung an eine heimische Umwelt. Auf der anderen Seite beruhe aber diese Politik der Angst darauf, der eigenen Heimlichkeit in der 
Unheimlichkeit des Anderen und Fremden zu begegnen, sie mithin immer erneut entfachen zu müssen (siehe Plessner 1981d, 193). Damit entgleitet diese Politik in eine Eigendynamik, die nicht mehr den eigenen Interessen dient. Durch die Festlegung von sich selbst darauf, Freund des Freundes und Feind des Feindes zu seinen, verliert man alle anderen Handlungsfreiheiten, bis einem am Ende die physischen Kräfte im Krieg ausgehen (siehe Plessner 1982g).

Die weltgeschichtliche Diagnose der globalen Ablösung des Westens vom Westen während seines Sieges darf Aktualität beanspruchen. Die Zukunft hängt davon ab, wie politisch und kulturell auf diese Herausforderung geantwortet wird, sei es eher in der Richtung der Totalisierung von Freund-FeindVerhältnissen, sei es eher in der Richtung gewaltenteiliger Ordnungsrahmen. Am Ende bleibt bei Plessner die Frage nach dem Menschen auch in ihrer geschichtsphilosophischen Fundierung offen, nicht aus einem frommen Wunsche, sondern als Einsicht in seine geschichtliche Wirklichkeit. Die naturphilosophische Fundierung legt den wesentlichen Struktur-Bruch zwischen der Exund der Re-Zentrierung frei, den es im personalen Leben als ständige Aufgabe zu verschränken gilt. Die sozial- und kulturphilosophische Fundierung beantwortet diese Aufgabe der künstlichen, vermittelten und utopischen Verschränkung durch Freilegung derjenigen dynamischen Strukturen, die die Personalität des Lebens praktisch ermöglichen, vom Spiel in und Schauspiel mit Personenrollen bis zu deren Verteilungen und dessen Grenzen. Die geschichtsphilosophische Fundierung legt die Ablösbarkeit der funktionalisierbaren Resultate und Methoden von derjenigen Gesellschaft und Kultur frei, in denen erstere ursprünglich erzeugt worden sind. Damit lösen sich alle personalen Zuordnungen, die praktisch eingerichtet worden waren, in die offene Frage nach einer anderen Zukunft auf. Nach dem Westen ist nun wohl der Osten weltgeschichtlich an der Reihe. Kann es ihm besser gelingen?

\section{Ausgewählte Vergleiche der Philosophischen Anthropologie mit anderen Philosophien}

Im vierten und letzten Teil des vorliegenden Buches wird die philosophische Spezifik dieser Philosophischen Anthropologie durch Vergleiche mit anderen Philosophien weiter herausgearbeitet. Zunächst lässt sich in der deutschen und europäischen Philosophietradition kaum ein größerer Gegensatz als der zwischen Kants Vernunftphilosophie und Nietzsches Lebensphilosophie denken. Plessners Vorgehen ist darauf ausgerichtet, die jeweils andere Philosophie derart immanent in sich selber zu kritisieren, dass sie aus sich heraus geführt und dem Anderen ihrer selbst geöffnet wird. Für ihn hat die lebensphilosophische 
Kritik nicht einfach die Vernunftphilosophie überwunden, sondern den Blick dafür frei gemacht, letztere anders als nur formal und dualistisch lesen zu können. Plessner setzt an der systematisch integrierenden Rolle der Urteilskraft in Kants Philosophie an, um sie über sich hinauszuführen. Sie kann so nicht nur im historischen Lebensprozess situiert werden, sondern auch als Muster für die philosophische Welterkenntnis von Personen reformuliert werden. Zwischen den modernen Forschungsunternehmen besteht kein gerichtlich auflösbarer Widerspruch, sondern ein Widerstreit, der der Bewahrung der Würde personaler Lebewesen bedarf. Auch in Nietzsches Philosophie entdeckt Plessner einen immanenten Hebel, nämlich den der Souveränität im europäischen Geist. Diese freigiebige Selbstüberwindung aller geschichtlichen Zurechnungen auf die eigene Schöpferkraft des Menschen befreit ihn von seinem Zirkel zu allen möglichen Selbstermächtigungen. Nietzsches Souveränität neuer Weltgewinnung überstrahlt so interpretiert seine Anthropo-Genealogie der Triebe, Verdrängungen, Sublimierungen und Ressentiments von Anthropoiden, in der sich seine Wirkungsgeschichte verkehrt hat.

Die Existenzphilosophie macht die Unvertretbarkeit und Einzigartigkeit des Menschen in seinem freien Tun stark gegen die anonymen Verhaltenszwänge in der Massengesellschaft und deren konformistische Ausreden. Ihre therapeutische Besinnung auf das Private und die je individuelle Innenwelt kann zum Asyl oder zur Regeneration für einen erneuten Einsatz in der Öffentlichkeit der Außen- und Mitwelt werden. Bei Jaspers geht sie von Grenzsituationen aus, die die Lebensführung erschüttern. Deren funktionales Pendant liegt bei Plessner, der mit Jaspers einen „ärztlichen Blick“ (Foucault) teilt, in den Lebenssituationen des ungespielten Lachens und Weinens vor. Was Plessner nicht ausführt, nur indirekt anzeigt, darin liegt Jaspers' Stärke: in der Erhellung spezifisch existenzieller Kommunikation und Liebe als Kampf mit einem für mich unvertretbaren und daher mein Selbst bildenden Du. Während Jaspers die Vernunft nicht durch die Existenz ersetzt wissen, sondern zum Ausgleich bringen wollte, radikalisierte Heidegger die Existenz phasenweise, zunächst individuell, dann gemeinschaftlich und schließlich seinsgeschichtlich. Demgegenüber fundierte Plessner die Existenz im Lebensprozess vom Standpunkt einer leiblich und geistig teilbaren Mitwelt. Gleichwohl gibt es, bei allen Gegensätzen, strukturell und funktional auch vergleichbare Problemlagen in Heideggers und Plessners Philosophien, die man umso besser erkennt, wenn man von Max Schelers (gegenüber Edmund Husserl) neuer Phänomenologie ausgeht. Schelers ordo amoris enthält Drittheiten, die aus den üblichen Fehlalternativen des EntwederOder hinausführen, insbesondere die Figur der Ek-stasis, die Plessner und Heidegger gegensätzlich transformieren. In der heutigen Diskussion fehlt dieser 
systematische Transformationszusammenhang völlig, was zu Fehlzuschreibungen (bei G. Agamben, J. Derrida, H. Schmitz und P. Sloterdijk) geführt hat.

Die letzten beiden Kapitel stellen einen systematischen Vergleich der Problemlage in den Philosophien von John Dewey und Helmuth Plessner an, zunächst in deren Naturphilosophien, sodann in ihren politischen Philosophien. Beide haben unabhängig voneinander die zentrale Frage behandelt, welcher neuen Aufgabe sich die Philosophie stellen muss, wenn die bis heute andauernde Wirkungsgeschichte Darwins stimmen sollte. Nehmen wir an, die Annahme sei richtig, dass die geistig-kulturelle Lebensform von Menschen in der Natur entstanden ist und sich auch künftig in der Natur halten können muss. Dann gilt es, die Natur neu zu denken, allerdings nicht so, wie sich dies Physikalismus und Biologismus vorstellen. Die Aufgabe besteht dann vielmehr darin, Natur so neu zu entwerfen, dass sie die irreduziblen Qualitäten von Leben und Geist wirklich ermöglichen kann. Diese wirkliche Ermöglichung von geistigem Leben schließt es gerade aus, die Natur auf ihre Rolle als Materie und Physis zu beschränken. Umgekehrt kann dann auch nicht der Mensch die Rolle Gottes unabhängig und außerhalb von der Natur übernehmen. Stattdessen wird zwischen drei „plateaus“ (Dewey) oder „Stufen“ (Plessner) unterschieden, zwischen der physikalischen (anorganischen), psycho-physischen (lebendigen) und der geistig lebenden Natur. Diese Unterscheidung wird so getroffen, dass ein Zusammenhang zwischen den Plateaus wirklich möglich wird. Die dritte Ebene, die geistige Lebensform, antworte geistig im Verhalten auf ihren Bruch mit den beiden anderen Ebenen, an die sie zugleich gebunden bleibt. Die geistige Lebensform sei daher auch künftig auf die stets erneute Differenz (zwischen Prekärem und Stabilem: Dewey; zwischen Unmittelbarem und Vermitteltem: Plessner) in der Erfahrung von Natur im neuen Sinne angewiesen. In der geistigen Lebensform bleibe die Natur im Ganzen eine offene Unbekannte, die die geistige Lebensform infrage stellt. Wenn aber die Natur im neuen Sinne nicht die Antwort aller Antworten, d. h. Antwortlichkeit, sondern die Frage aller Fragen, d. h. Fraglichkeit, darstellt (Krüger 2006b), dann bedarf die moderne Gesellschaft einer neuen öffentlichen Gewaltenteilung und zivilgesellschaftlicher Initiativen, um die Verhältnisse zur äußeren und eigenen Natur grundsätzlich zu transformieren. Statt geistige Kultur durch immer neue technische Reduktionen abzuwerten, geht es um ihre Erneuerung in der öffentlichen Kommunikation zwischen den Laien- und Expertenkulturen. In der gleichzeitig ökologischen, kapitalökonomischen und politischen Krise ist sie das Entwicklungspotential zum Neuanfang. 
